\title{
Number Zero property in factoring a number into prime factors ( Addition of Zeros)
}

Author: Alessandro Boatto Jesolo lido (VE), 29 July 2019

\begin{abstract}
This property of prime numbers is based on the addition of a number of zeros between a prime number $(P)$ and the same prime number $(P)$ to form a compound number usually always, except in rare cases, from three prime factors where two factors are always the prime number 11 , the first number ( $P$ ) and a third prime number which is always $9090 . .9091$ (ends with the number 91 but the initial number 90 repeats but depends on the size of prime number $(P)$. The amount of Zeros to be added is equal to the number of digits of the prime number $(P)$. If I take the prime number 43 for the assumption which has two digits have to add three zeros and the number becomes 4300043 .
\end{abstract}

$$
(P)+\text { Zero + ( P ) }
$$

\section{Content}

I demonstrate with two examples that the number obtained from the formula ( $P)+$ Zeros $+(P)$, you have to divide with the number 11 and with the number $(P)$ to get the first factor 90 . 9091 .

\section{Example 1}

No. 43

$\% 1=43$

? 4300043

$\% 2=4300043$

? $\% 2 / 11$

$\% 3=390913$

? \%3/\%1

$\% 4=9091$

\section{Example 2}

No. 
$\% 1=$

19008712816648221131268515739354139754718967899685154936666385390880271038021 04498957191261465571

$<71896789968515493666638539088027103802104498957191261465571$

$\div 2=$

19008712816648221131268515739354139754718967899685154936666385390880271038021 04498957191261465571000000000000000000000000000000000000000000000000000000000 00000000000000000000000000000000000000000190087128166482211312685157393541397 5471896789968515493666638539088027103802104498957191261465571

$? \% 2 / 11$

$\% 3=$

17280648015134746482971377944867399777017243545168322669696713991709337307291 85908142901146786882727272727272727272727272727272727272727272727272727272727 27272727272727272727272727272727272727272900079207424074737556986506721401270 497445162724410499424239867189820645800191318087017387405961

? $\% 3 / \% 1$

$\% 4=$

90909090909090909090909090909090909090909090909090909090909090909090909090909 09090909090909090909090909090909090909090909090909090909090909090909090909090 9090909090909090909090909090909090909091

\section{Conclusions}

This article wants to highlight this property of the many prime numbers that I have tested and would like to give a rational demonstration in the future. 